Bot. Mag. Tokyo 84 18-23 (January 25, 1971)

\title{
Chromocenters in Rumex acetosa nucleus
}

\author{
by Masahide KURITA* and Yûzô KUROKI*
}

Received November 13, 1970

\begin{abstract}
A study was made on chromocenters including sex chromatin bodies in somatic nuclei of Rumex acetosa $\mathrm{L}$. At mid-prophase in male clone used for study, two Y-chromosomes consisting of a heterochromatin are more deeply stained through entire length than any other chromosome and have their well-defined outlines. Two X-chromosomes and autosomes of female clone are similar in a figure of mid-prophase to the X-chromosome and the autosomes of male clone respectively.

Male nuclei are classified into four types according to chromocenters: the first type presents no chromocenter, the second has only small chromocenters varying in size, the third has one large and some small chromocenters, and the fourth has two large and some small chromocenters. The large chromocenters are certain to have been originated from Y-chromosomes, of which a segment occasionally reveals itself as a small chromocenter as known from the second typed nucleus. The number of chromocenter per nucleus is from zero to about ten, and the nuclei with about five or six chromocenters are of the most frequent occurrence. Thus, the chromocenters, not to mention those ( $=$ sex chromatin bodies) originated from Y-chromosomes, vary in number and size with a cell, viz. a phenomenon of heteropycnosis is not to be stable.

In almost all cases, female nucleus presents no chromocenter in contrast with male one which has two Y-chromosomes consisting of heterochromatin and which presents most often five or six chromocenters. This may show that the heterochromatin has a tendency to gather an euchromatin into a mass which must be treated as chromocenter.
\end{abstract}

\section{Introduction}

A number of papers were already published on a Barr body or a sex chromatin body, together with or without a chromocenter, since a sexual dimorphism of nucleus was found by Smith (cited from Mittwoch ${ }^{1)}$ ) and Barr and Bertram ${ }^{2}$. Those papers show that, varying with a cell, the Barr body is not always stable. According to Schwarzacher ${ }^{3}$, the body was found in $46 \%$ of nuclei in living cells and in $90 \%$ of nuclei in cells after fixation and staining. Mittwoch et al. ${ }^{4)}$ noted that the Barr body was rarely seen in cells with large nuclei in contrast with the other cells having usually the body. Graham and $\operatorname{Barr}^{5)}$ reported that a sex chromatin body appeared in one-half to two-thirds of the cells in certain tissues from a female cat. By Moore and $\mathrm{Barr}^{6)}$ it was described that a chromocenter was smaller than the Barr body and had a less well-defined outline.

A study was made on sex chromatin of Rumex acetosa L. by Shimizur). In male nucleus, the chromatin was shown as a darkly stained body which was not found in female nucleus. The present authors ${ }^{8)}$ have made an observation on condensation

\footnotetext{
* Biological Institute, Ehime University, Matsuyama, Japan.
} 
of Y-chromosomes at somatic prophase of the species. Certain segment in its chromosome-arm has been found to originate from a chromocenter. Reports on the chromocenter including a sex chromatin body in the species are, however, a few and seem not to be detailed enough. A study has been carried out on the chromocenters by the present authors. The result will be given in this paper.

\section{Material and Method}

The material used is three female and four male clones of Rumex acetosa as follows: 우) clone 74 (C-74) collected from Morioka City, clone 106 (C-106) from Kurayoshi City, clone 128 (C-128) from Hokkaidô, $\hat{\text { o) }}$ clone 84 (C-84) from Shiraishi City, clone 110 (C-110) from Kurayoshi City, clone 124 (C-124) from Hokkaidô, clone 274 (C-274) from Miyako City. In all the clones, ten large autosomes are of subterminal type and two small ones are of submedian type (Fig. 1, 3, 5, 6). At almost all mid-prophases, the $\mathrm{Y}$-chromosomes are more compact than any other chromosome and their outlines are bolder (Fig. 2,4).

Cytological preparations were made from root tips following the same method as employed in our early study (Kuroki and Kurita ${ }^{9)}$ ). In many cases, the procedure for male materials was carried forward in parallel with that for female ones. Chromocenters were counted in nuclei which were heavily pressed to disturb them slightly in outlines.

\section{Observation}

1. Male nucleus

Chromocenters are variable in morphology and there are a few cells whose

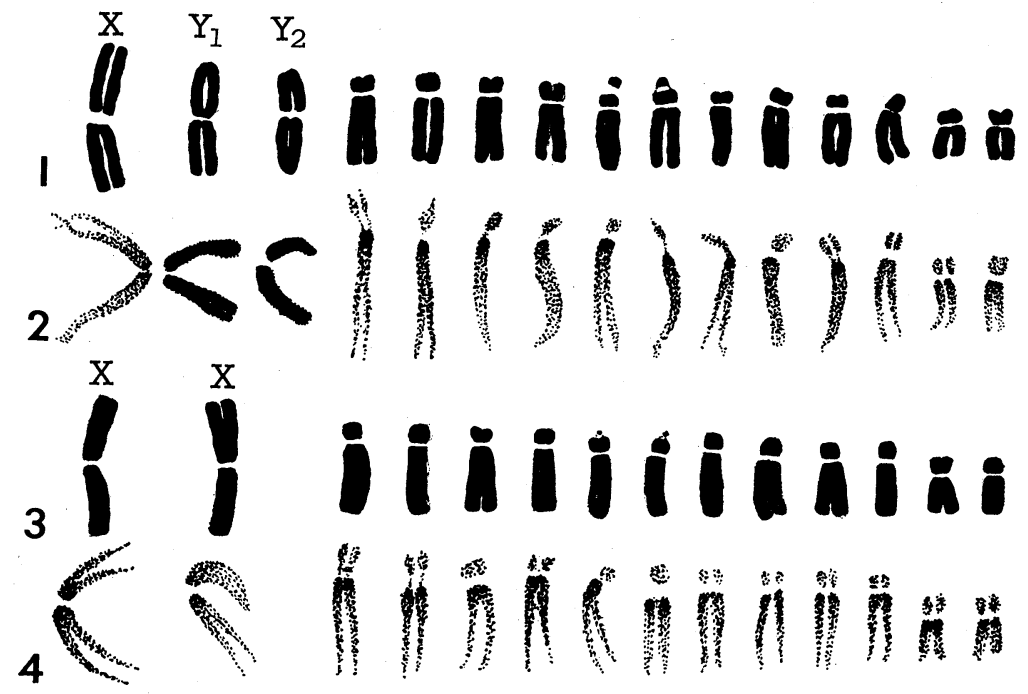

Figs. 1-4. Somatic chromosomes at metaphase in C-110 (Fig. 1), mid-prophase in C124 (Fig. 2), metaphase in C-74 (Fig. 3) and mid-prophase in C-106 (Fig. 4). Figs. 1 and 2 : male, Figs. 3 and 4 : Female. $\times 2180$. 


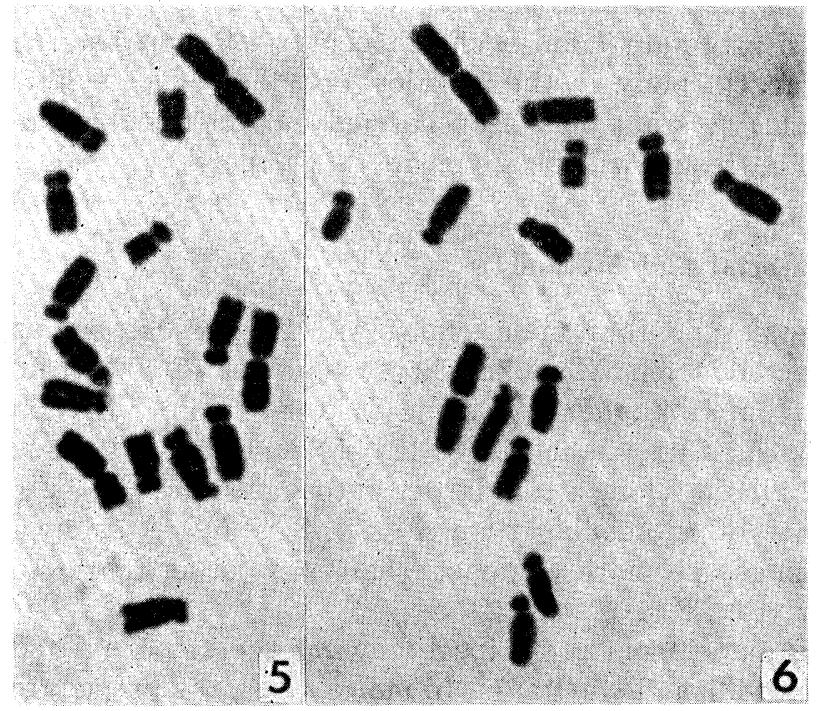

Figs. 5 and 6 . Photomicrograph of metaphase chromosomes in male C-274 (Fig. 5) and female C106 (Fig. 6). $\times 1700$. nuclei present no chromocenter. Figs. 8 and 13 show that all the chromocenters in single nucleus are small, being about $0.8 \mu$ to $1.5 \mu$ in length and are spherical or ellipsoidal. Some chromocenters (Fig. 14b) are dumb-belled in shape. Small chromocenters are generally compact and homogenous. There is also found a chromocenter which is split into two halves or which is empty at the core as mentioned already (Kurita and Kuroki $\left.^{8}\right)$. Large chromocenter which is about $3.7 \mu$ to $4.4 \mu$ in length is not homogenous in almost all cases (Fig. 14a, 16a, 16b). Some segments in the chromocenter are compact and homogenous, but the others are observed to be a

fairly loose gathering of minute chromatin granules. As shown in Figs. 14a and $16 \mathrm{~b}$, some large chromocenters are considered to consist of three compact and homogenous segments and two coarse segments presenting minute granules. At present, it remains questionable whether this pattern is fundamental for all the large chromocenters or not. There is sometimes found a nucleus (Fig. 9, 10) in which the large chromocenter is not two but one in number. In a very few nuclei, there appears the large chromocenter (Fig. 12a, 15a) which is closely similar in shape and in condensation to $\mathrm{Y}_{2}$-chromosome at metaphase. But the chromocenter is fairly smaller in size than the $\mathrm{Y}_{2}$ (cf. Figs. 1 and 15, Figs. 5 and 12), though it is judged from the base of a few measurements.

It is difficult to decide the number of chromocenter per nucleus as often stated already. The present authors take count of the chomocenter in heavily pressed nuclei which are suitable for counting. Table 1 shows the chromocenter numbers which are considered to be sure or nearly sure. The number varies from zero to ten. It may be admitted that, in the male clones used fort he present study, the number of chromocenter per nucleus is roughly five to six.

\section{Female nucleus}

Fig. 7 shows one out of many nuclei having no chromocenter. In some nuclei, the authors have recognized a few compact bodies which are appropriate to be treated as a chromocenter. These bodies are always small. There is no body which is similar in size to the large chromocenter found in male nucleus. The result of chromocenter count in female nuclei is also shown in Table 1. Generally speaking, it may be safe that there occurs no chromocenter in the nuclei from the female clones used for the present study. 


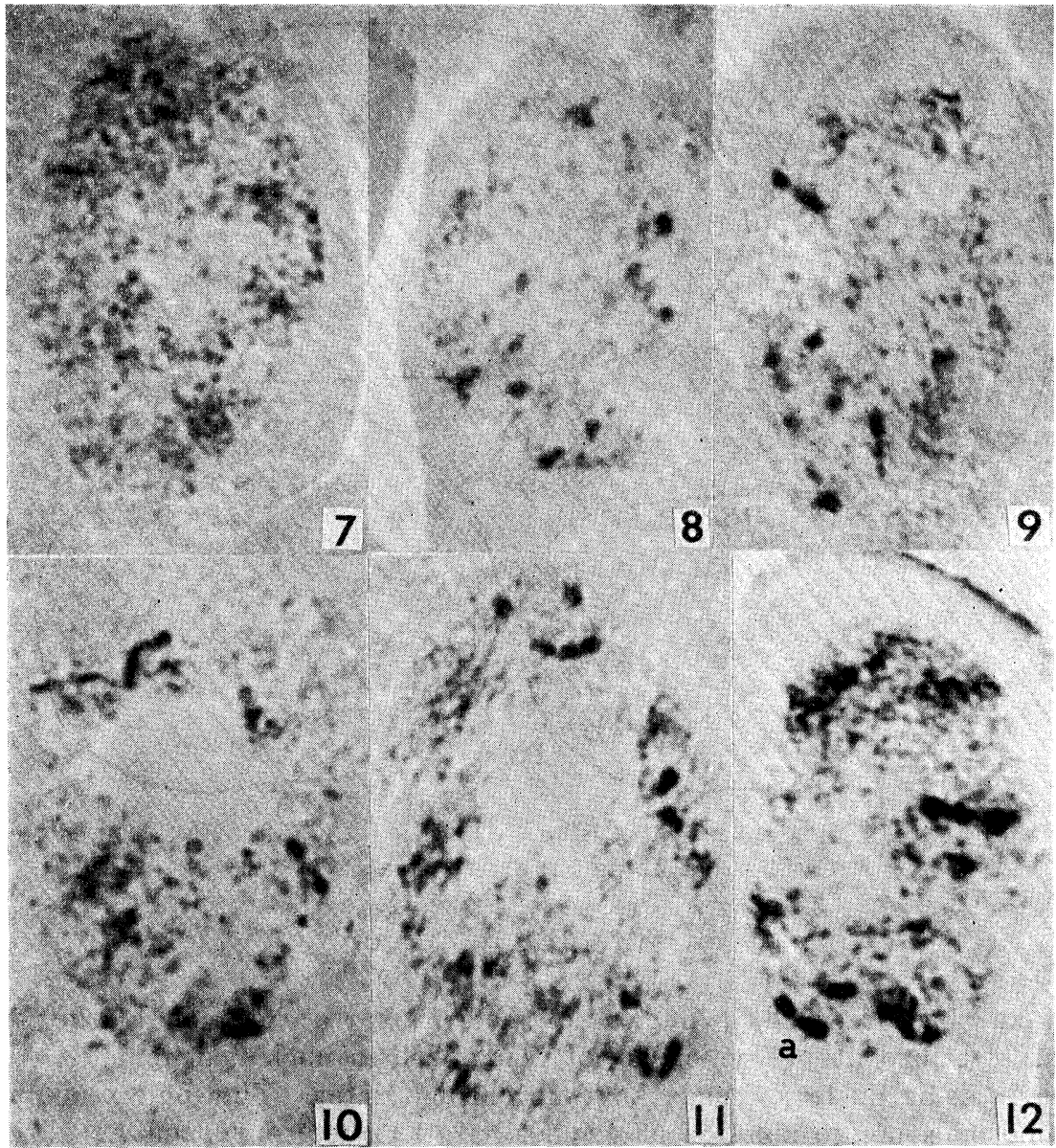

Figs. 7-12. Photomicrograph of interphase nucleus in C-74 (Fig. 7), C-124 (Fig. 8), C-110 (Figs. 9 and 10) and C-84 (Figs. 11-12). Fig. 7: Female, Figs. 8-12: male. $\times 1700$.

Table 1. Calculation of chromocenters.

\begin{tabular}{|c|c|c|c|c|c|c|c|c|c|c|c|c|c|}
\hline \multirow{2}{*}{ Sex } & \multirow{2}{*}{ Clone No. } & \multicolumn{12}{|c|}{ No. of chromocenter per nucleus } \\
\hline & & 0 & 1 & 2 & 3 & 4 & 5 & 6 & 7 & 8 & 9 & 10 & 11 \\
\hline \multirow{4}{*}{$\hat{\delta}$} & 84 & 3 & 2 & 9 & 9 & 14 & 24 & 18 & 18 & 11 & 5 & 2 & 0 \\
\hline & 110 & 5 & 4 & 16 & 28 & 32 & 30 & 32 & 13 & 10 & 4 & 0 & 0 \\
\hline & 274 & 4 & 3 & 3 & 10 & 16 & 20 & 22 & 26 & 11 & 10 & 4 & 0 \\
\hline & $\begin{array}{l}\text { Total number } \\
\text { of nuclei }\end{array}$ & 12 & 9 & 28 & 47 & 62 & 74 & 72 & 57 & 32 & 19 & 6 & 0 \\
\hline \multirow{3}{*}{ 우 } & 74 & 160 & 8 & 1. & 0 & 0 & 0 & 0 & 0 & 0 & 0 & 0 & 0 \\
\hline & 106 & 200 & 19 & 5 & 2 & 0 & 0 & 0 & 0 & 0 & 0 & 0 & 0 \\
\hline & $\begin{array}{l}\text { Total uumber } \\
\text { of nuclei }\end{array}$ & 360 & 27 & 6 & 2 & 0 & 0 & 0 & 0 & 0 & 0 & 0 & 0 \\
\hline
\end{tabular}




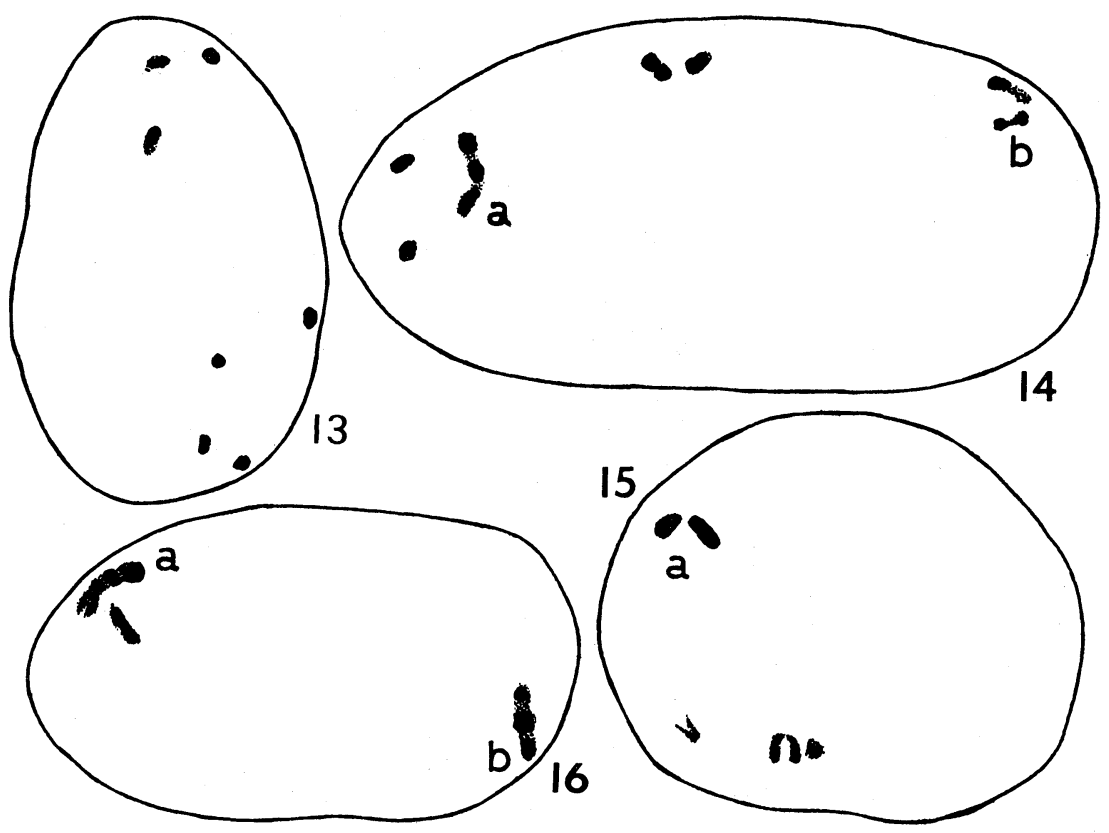

Figs. 13-16. Interphase nucleus in C-110 (Fig. 13), C-84 (Fig. 14) and C-274 (Figs. 15 and 16). All: male. $\times 2180$.

\section{Discussion}

Shimizu ${ }^{7)}$ has observed, in nucleus of male Rumex acetosa, one large sex-chromatin mass stained deeply which is not recognized in nucleus of female. As shown in Table 1, some nuclei of male have no chromocenter. This points out that any sex-chromosome, not to mention autosomes, is of no heteropycnosis at all in those nuclei.

The authors ${ }^{8)}$ have found that a chromocenter reveals itself afterwards as an outer segment in arm of Y-chromosome at metaphase. As found in Figs. 8 and 13, there are male nuclei in which all the chromocenters, being about $0.8 \mu$ to $1.5 \mu$ in length, are much smaller than the Y-chromosomes. Judging from this observation, it can be also said with safety that the Y-chromosomes are not to be entirely but partially heteropycnotic as mentioned already by the authors. ${ }^{8)}$

Further, there are some male nuclei (Fig. 10) which have one large chromocenter measuring about $3.7 \mu$ to $4.4 \mu$ in length besides some small chromocenters, and the other male nuclei (Fig. 16) which have two of such large one. The large chromocenters are considered to originate from Y-chomosomes, because those chromocenters are found in nucleus of male and never in nucleus of female. The nucleus presenting one large chromocenter shows that there has arisen a sharp size-difference in heteropycnosis between the two Y-chromosomes by the cause of inner condition in the nucleus.

As shown in Figs. 12a and 15a, there is rarely observed a chromocenter which is closely similar in shape and in staining to the metaphase Y-chromosome and which is fairly smaller than the $\mathrm{Y}$. This chromocenter is considered to be originated from the entire chromatin of the Y-chromosome and to be smaller because of 
having no matrix. Then, the $\mathrm{Y}$-chromosome in this case may be said to be entirely heteropycnotic.

As noted by the earlier studies on an animal (Schwarzacher ${ }^{3)}$, Mittwoch et $a ._{.}^{4}$, and so on), the chromocenters in single cell of Rumex acetosa are also variable in size and in number, namely heteropycnosis varies with a cell.

As shown in Figs. 2 and 4, male clones are not different in a figure of midprophase chromosomes from female clones with the exception of two Y-chromosomes. These Y's are generally stained more deeply than any other chromosome and are bolder in their outlines. Each of X-chromosomes in male and in female is fairly stained at both sides adjacent to centromere and all the autosomes in both sexes are similarly at the proximal ends of long arms. Now, Table 1 shows that the chromocenters per nucleus are much more in male nucleus than in female one. Then, the heterochromatin in Y-chromosomes is considered to have a tendency to gather an euchromatin into a mass which must be treated as a chromocenter.

\section{References}

1) Mittwoch, U., Sex chromosomes, Academic press, New York (1967).

2) Barr, M.L. and Bertram, E. G., Nature $163: 676$ (1949).

3) Schwarzacher, H. G., Cytogenet. 2: 117 (1963).

4) Mittwoch, U., Lele, K.P. and Webster, W.S., Nature $205: 477$ (1965).

5) Graham, M.A. and Barr, M. L., Anatom.
Rec. 112: 709 (1952):

6) Moore, K.L. and Barr, M. L., Acta Anat. 21 : 197 (1954).

7) Shimizu, Y., Kromosomo 49 : 1521 (1961).

8) Kurita, M. and Kuroki, Y., Japan. J. Genet. 45 : 255 (1970).

9) Kuroki, Y. and Kurita, M., Mem. Ehime Univ., Sci. Ser. B, 6 : 41 (1969). 